\title{
KOMPARASI KLASIFIKASI PADA PREDIKSI PENDAPATAN RUMAH TANGGA
}

\author{
Evy Priyanti \\ Program Studi Sistem Informasi Akuntansi \\ Universitas Bina Sarana Informatika (UBSI) \\ JI. Kamal Raya No 18 Ringroad Barat, Cengkareng, Jakarta Barat \\ evy.evp@bsi.ac.id
}

\begin{abstract}
ABSTRAK
Kebutuhan akan kehidupan sehari-hari tidak terlepas dari pendapatan yang dihasilkan, baik pendapatan harian, mingguan atau bulanan. Oleh karena itu prediksi pendapatan rumah tangga sangat penting dikarenakan akan membantu dalam menciptakan pendapatan yang lebih baik, dalam memprediksi suatu data dapat dilakukan dengan beberapa algoritma diantaranya dengan algoritma Knearest neighbor dan algorima Neural Network. Pada penelitian kali ini akan dikomparasi bagaimana algoritma K-Nearest neighbor dengan Neural network dalam memprediksi pendapatan rumah tangga pada sensus yang dilakukan di Bereu pada tahun 1996. Algoritma K-Nearest neighbor menghasilkan nilai akurasi sebesar $70,49 \%$ sedangkan algoritma Neural Network menghasilkan akurasi sebesar $83,62 \%$, hal ini membuktikan bahwa algoritma Neural Network dapat bekerja lebih baik dalam memprediksi pendapatan rumah tangga di Bereu yang dilakukan oleh Ronny Kohavi dan Barry Becker pada 1 mei 1996 yang terdiri dari 48842 dataset dan 14 atribut. Beberapa atribut menjadi faktor penentu dalam menciptakan pendapatan yang lebih tinggi, diantaranya status pernikahan yang utuh yang terdiri dari minimal suami dan istri dalam satu atap dan pendidikan yang tinggi mendapatkan peluang untuk mendapatkan penghasilan yang lebih tinggi dari pasangan itu sendiri yang nantinya dapat mempengaruhi pendapatan rumah tangga lebih dari $\$ 50.000 /$ tahun. Selain itu faktor pengalaman bekerja juga menjadi salah satu faktor penentu tingginya pendapatan rumah tangga. Faktor ketidakharmonisan dalam rumah tangga juga menjadi salah satu fakto pendapatan yang kurang dari $\$ 50.000 /$ tahun.
\end{abstract}

\begin{abstract}
The need for daily life is inseparable from the income generated, whether daily, weekly or monthly income. Therefore prediction of household income is very important because it will help in creating a better income, in predicting a data can be done with several algorithms including the K-nearest neighbor algorithm and Neural Network algorithm. This study will compare how the K-Nearest neighbor algorithm with Neural Network predicts household income in the census conducted in Bereu in 1996. The K-Nearest neighbor algorithm produces an accuracy value of $70.49 \%$ while the Neural Network algorithm produces an accuracy of $83.62 \%$, this proves that the Neural Network algorithm can work better in predicting household income in Bereu conducted by Ronny Kohavi and Barry Becker on May 1, 1996 consisting of 48842 datasets and 14 attributes. Some attributes are the deciding factors in creating higher income, including complete marital status consisting of a minimum of husband and wife under one roof and higher education to get opportunities to earn higher income from the couple themselves which can later affect household income more than \$50,000/year. In addition, work experience is also one of the determinants of high household income. The disharmony factor in the household is also a fact that income is less than $\$ 50,000 /$ year.
\end{abstract}

Keyword :Household,.Neural Network, K-NN 


\section{Pendahuluan}

Sensus yang dilakukan oleh Ronny Kohavi and Barry Becker pada 1 mei 1996 ini terdiri dari 48842 data. Sensus ini dilakukan di Bereu didapatkan data pendapatan untuk beberapa Negara di dunia dengan besaran yang sudah diklasifikasikan menjadi beberapa bagian yaitu pendapatan yang lebih besar dari $\$ 50.000 /$ tahun, pendapatan yang sama besar $\$ 50.000 /$ tahun dan pendapatan yang kurang dari $\$ 50.000 /$ tahun. Data yang didapat terdiri dari beberapa atribut yaitu age, workclass, state-gov, fn/wgt, education, education-num, marital-status, occupation, relationship, race, sex, capital-gain, capital-loss, hours-per-week, native-country .(Priyanti, 2017)

Beberapa faktor yang mempengaruhi pendapatan rumah tangga lebih dari $\$ 50.000 /$ tahun diantaranya adalah jenis keluarga, bahwasannya keluarga yang utuh yang minimal terdiri dari suami dan istri dalam satu atap memiliki peluang mendapatkan pendapatan rumah tangga yang lebih besar dibandingkan dengan jenis keluarga yang tidak utuh. Pendidikan, seseorang yang memiliki pendidikan yang lebih tinggi memiliki peluang untuk mendapatkan penghasilan yang lebih tinggi. Lama bekerja, semakin lama seorang pekerja semakin besar pula penghasilan yang didapatkannya dan ini membuktikan bahwa seseorang dengan pengalaman yang lebih banyak dapat menghasilkan pendapatan yang lebih banyak pula. (priyanti,2017).

Pada penelitian kali ini akan dibuktikan bahwa algoritma neural network akan meningkatkan akurasi dari data pendapatan penduduk ada di Bereu.

Menurut Heaton (2010:p27) Neural Network adalah jaringan saraf yang mensimulasikan jaringan saraf biologis manusia kedalam arsitektur komputer dan arsistektur algoritma baru terhadap komputer konvensional. Hal ini memungkinkan penggunaan operasi komputasi (penambahan, pengurangan, dan elemen logika fundamental) yang sangat sederhana untuk memecahkan masalah yang kompleks, matematis yang tidak jelas, masalah nonlinear atau masalah stokastik.

Penelitian sebelumnya Valentin Mihov dalam penelitiannya yang berjudul Adult Income Dataset Analysist with IPython menggunakan Learning Model Logistic regression pada tahun 2015 yang hanya memisahkan antara data yang berhubungan dengan data yang tidak berhubungan. Fitur yang bertanda negatif terdiri dari Never married, Own child, priv-house-serv dan divorce sedangkan fitur yang bernilai positif terdiri dari capital gain, married-civ-spouse, age, hours per week, dan exec-managerial, selanjutnya fitur yang bernilai negatif akan dipisahkan supaya proses analisis dapat berjalan lebih baik.

\section{Metode Penelitian}

Atribut-atribut yang digunakan pada penelitian kali ini didapat dari UCI dataset yang terdiri dari :

age: dengan jenis continuous.

workclass: terdiri dari Private, Self-emp-not-inc, Self-emp-inc, Federal-gov, Local-gov, Stategov, Without-pay, Never-worked.

fnlwgt: dengan jenis continuous.

education: terdiri dari Bachelors, Some-college, 11th, HS-grad, Prof-school, Assoc-acdm, Assoc-voc, 9th, 7th-8th, 12th, Masters, 1st-4th, 10th, Doctorate, 5th-6th, Preschool.

education-num: dengan jenis continuous.

marital-status: terdiri dari Married-civ-spouse, Divorced, Never-married, Separated, Widowed, Married-spouse-absent, Married-AFspouse.

occupation: terdiri dari Tech-support, Craftrepair, Other-service, Sales, Exec-managerial, Prof-specialty, Handlers-cleaners, Machine-opinspct, Adm-clerical, Farming-fishing, Transport-moving, Priv-house-serv, Protectiveserv, Armed-Forces.

relationship: terdiri dari Wife, Own-child, Husband, Not-in-family, Other-relative, Unmarried.

race:terdiri dari White, Asian-Pac-Islander, Amer-Indian-Eskimo, Other, Black.

sex: terdiri dari Female, Male.

capital-gain: dengan jenis continuous.

capital-loss: dengan jenis continuous.

hours-per-week: dengan jenis continuous.

native-country: terdiri dari United-States, Cambodia, England, Puerto-Rico, Canada, Germany, Outlying-US(Guam-USVI-etc), India, Japan, Greece, South, China, Cuba, Iran, Honduras, Philippines, Italy, Poland, Jamaica, Vietnam, Mexico, Portugal, Ireland, France, Dominican-Republic, Laos, Ecuador, Taiwan, Haiti, Columbia, Hungary, Guatemala, Nicaragua, Scotland, Thailand, Yugoslavia, ElSalvador, Trinadad\&Tobago, Peru, Hong, Holand-Netherlands.

Atribut-atribut diatas dideskripsikan sebagai berikut :

Usia terdiri dari usia 17-90 tahun.

Kelas pekerja terdiri dari pegawai swasta, wiraswasta yang bukan dari perusahaan, pemerintah federal, pemerintah local, pemerintah pusat, pekerja tanpa bayaran, dan tidak pernah bekerja.

Sample adalah nomor sample

Pendidikan terdiri dari Sarjana, Beberapa perguruan tinggi, kelas 11, HS-grad, Prof- 
school, Assoc-acdm, Assoc-voc, 9th, 7th-8th, 12th, Masters, 1st-4th, 10th, Doctorate, 5th-6th, Prasekolah

Nomor pendidikan terdiri dari 1 sampai dengan 16.

Status pernikahan terdiri dari Married-civspouse=menikah dengan warga sipil, Divorced=bercerai, Never-married=memiliki pasangan tetapi tidak menikah secara resmi, Separated=terpisah, Widowed=janda atau duda yang belum menikah lagi, Marriedspouse-absent=pasangan yang menikah akan tetap tidak satu rumah tangga bisa jadi karena dipisahkan atau belum resmi bercarai akan tetapi sudah tidak 1 rumah, Married-AFspouse $=$ menikah dengan angkatan/prajurit.

Jenis keluarga juga terdiri dari dua bagian yaitu keluarga lengkap dan keluarga tidak lengkap. Yang terdiri dari keluarga tidak lengkap adalah divorce, never-married, separated, married spouse-absen

Keluarga lengkap terdiri dari married-civspouse dan married-AF-spouse.

Pekerjaan: Tech-support, Craft-repair, Otherservice, Sales, Exec-managerial, Profspecialty, Handlers-cleaners, Machine-opinspct, Adm-clerical, Farming-fishing,

Dikarenakan fungsi aktivasi pada neural network yang dipakai fungsi aktivasi biner, data harus ditransformasikan dulu karena batasan keluaran fungsi aktivasi sigmoid adalah $[0,1]$, tapi akan lebih baik jika ditransformasikan ke interval yang lebih kecil, missal pada interval Berikut perhitungan transform dengan menggunakan fungsi aktivasi sigmoid:

Berdasarkan data pendapatan rumah tangga dengan atribut age diketahui

nilai minimum $(a)=17$

nilai maximum $(b)=90$

pendapatan rumah tangga yang akan di transform $(\mathrm{x})=x^{l}=\frac{0.8(39-17)}{90-17}+0.1$

\section{Hasil dan Pembahasan}

Algoritma K-Nearest Neighbors dikenal sebagai algoritma lazy learning karena membutuhkan lebih sedikit waktu untuk pelatihan. Komputasinya seperti algoritma pembelajaran eager-based learning yang membutuhkan waktu lebih sedikit selama klasifikasi. (singh, 2017). Pelatihan dalam k-nn untuk memprediksi label kelas kategorikal (Nikam, 2015). Pada K-Nearest neigbour membutuhkan nilai $\mathrm{k}$ tetangga atau nilai $\mathrm{k}$ disekitarnya instance data uji baru untuk diklasifikasikan keladalam kelas maksimum, disarankan kelas $\mathrm{k}$ bernilai ganjil supaya akurasi klasifikasi dapat memperoleh nilai yang lebih tinggi (nurwanto, 2016).

Berikut algoritma k-nearest neigbour:
Transport-moving, Priv-house-serv, Protectiveserv, Armed-Forces.

hubungan: istri, anak kandung, suami, bukan bagian dari keluarga inti, relasi lainnya, tidak menikah.

ras: White, Asian-Pac-Islander, Amer-IndianEskimo, Other, Black.

jenis kelamin: wanita, pria.

keuntungan dalam bentuk uang: bernilai antara 1 sampai dengan 99.999 kehilangan modal: bernilai antara 1 sampai dengan 3.004 .

jam per minggu: bernilai antara 1 sampai dengan 99.

Negara asal: United-States, Cambodia, England, Puerto-Rico, Canada, Germany, Outlying-US(Guam-USVI-etc), India, Japan, Greece, South, China, Cuba, Iran, Honduras, Philippines, Italy, Poland, Jamaica, Vietnam, Mexico, Portugal, Ireland, France, DominicanRepublic, Laos, Ecuador, Taiwan, Haiti, Columbia, Hungary, Guatemala, Nicaragua, Scotland, Thailand, Yugoslavia, El-Salvador, Trinadad\&Tobago, Peru, Hong, HolandNetherlands.

[0.1,0.9] (Siang ,2009:p25). Maka, pada data pendapatan rumah tangga yang ada dilakukan transform data dengan interval $[0.1,0.9]$, dengan rumus sebagai berikut:

$x^{1}=\frac{0.8(22)}{73}+0.1$

$$
x^{l}=\frac{0.8(x-a)}{b-a}+0.1
$$

$x^{1}=0.889$

Selanjutnya akan dilakukan pengujian dan pelatihan apakah dengan nilai transform pendapatan rumah tangga dengan nilai atribut 39 dan nilai transform 0.889 memiliki nilai akurasi yang tinggi.

Calculate optimal $k$ with least misclassification error. Create empty list predictions

For each $t$ in testSet

Find neighbors:

Create empty list distances

For each $x$ in trainingset

dist= Euclidean distance measure between $x$ and test instance $t$

distances. append(training Set [x], dist)

End For

Sort distances in ascending order.

Create list neighbors by taking $k$ subset of training points from distances

Return neighbors 


\section{result $=$ maximum voted class in the neighbors \\ predictions.append(result) \\ End For \\ Calculate accuracy using predicted list predictions of target values and actual target values}

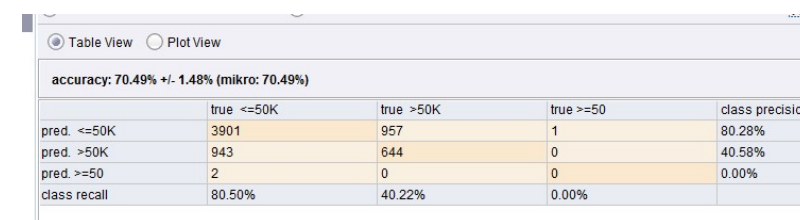

Gambar 3.1. Hasil Akurasi K-NN

Rule Model yang dihasilkan pada K-NN sebagai berikut :

if age $\leq 31.500$ then $\quad<=50 \mathrm{~K} \quad(2076$

/ 161 / 1)

if education-num $\leq 11.500$ and relationship $>3.500$ then $<=50 \mathrm{~K}$ $(566 / 25 / 0)$

if education-num $\leq 11.500$ and capital-gain $\leq 4243.500$ then $<=50 \mathrm{~K}$ $(1595 / 546 / 0)$

if capital-gain > 5095.500 then $>50 \mathrm{~K}(10 / 277 / 0)$

if marital-status $>2.500$ and sex $>$ 1.500 and fnlwgt $\leq 179660.500$ then $<=50 \mathrm{~K} \quad(80 / 5 / 0)$

if capital-loss > 1794.500 then $>50 \mathrm{~K}(6 / 88 / 0)$

if hours-per-week $\leq 42.500$ and fnlwgt $>\quad 206248.500$ and relationship $\leq 1.500$ and fnlwgt $\leq$ 305627.500 then $\quad=50 \mathrm{~K} \quad(36 / 4 /$ $0)$

if marital-status $\leq 2.500$ and hours-per-week $>41$ and maritalstatus $>1.500$ and occupation $\leq 3$ and hours-per-week $\leq 57.500$ then $>50 \mathrm{~K}(9 / 61 / 0)$

if marital-status $>2.500$ and education-num $\leq 12.500$ then $<=50 \mathrm{~K}$ $(27 / 1 / 0)$

if workclass $\leq 3.500$ and occupation $>4.500$ and age $\leq 40.500$ and hoursper-week $\leq 41$ and education-num $\leq$ 13.500 and fnlwgt > 224890 then $<=50 \mathrm{~K} \quad(11 / 2 / 0)$

if relationship > 1.500 and education $>1.500$ and age $>33.500$ and fnlwgt $\leq 202449.500$ and fnlwgt $>121054.500$ and fnlwgt $\leq 143877$ then >50K $(0 / 17 / 0)$

if education-num $\leq 14.500$ and hours-per-week $\leq 35.500$ and age > 54.500 and age $>61.500$ then $<=50 \mathrm{~K}$ $(17 / 3 / 0)$

if education-num $\leq 14.500$ and marital-status $>2.500$ and hours- per-week $\leq 44$ and workclass $>2.500$ and age $\leq 50$ then $\quad<=50 \mathrm{~K} \quad(22 / 1 /$ $0)$

if education-num > 14.500 and age $\leq$ 52.500 and fnlwgt $\leq 346399.500$ and fnlwgt > 106099.500 then >50K (5 / 41 / 0)

if workclass $\leq 3.500$ and nativecountry $>4.500$ and native-country $\leq 9.500$ then $<=50 \mathrm{~K} \quad(14 / 2 / 0)$

if workclass $\leq 3.500$ and hours-perweek > 58 and fnlwgt > 194906 and workclass $\leq 2.500$ then $\quad<=50 \mathrm{~K} \quad(9 /$ 2 / 0 )

if relationship $\leq 3.500$ and relationship > 2.500 and age s 37.500 then $>50 \mathrm{~K}(2 / 15 / 0)$

if relationship > 3.500 and marital-status $\leq 1.500$ then $<=50 \mathrm{~K}$ $(35 / 2 / 0)$

if fnlwgt > 119015 and relationship $>1.500$ and fnlwgt $\leq 201918$ and occupation $\leq 6.500$ and age $\leq 50.500$ and age > 40.500 then >50K (5 / $39 / 0)$

if fnlwgt $\leq 204424.500$ and age $\leq$ 47.500 and fnlwgt $>150182$ and age $>37.500$ and fnlwgt > 190655.500 then $\quad<=50 \mathrm{~K} \quad(12 / 0 / 0)$

if fnlwgt > 188539.500 and fnlwgt $\leq$ 243494 and age $\leq 46.500$ and age > 39.500 and workclass $>2.500$ then $>50 \mathrm{~K}(2 / 16 / 0)$

if capital-gain $>4518$ then $<=50 \mathrm{~K}$ $(18 / 1 / 0)$

if workclass > 3.500 and fnlwgt s 160164.500 and education-num > 13.500 and age $\leq 47.500$ then $>50 \mathrm{~K}$ $(0 / 8 / 0)$

if age $\leq 47.500$ and occupation $\leq$ 1.500 and fnlwgt $\leq 187078.500$ then $<=50 \mathrm{~K} \quad(18 / 1 / 0)$

if $\operatorname{sex} \leq 1.500$ and fnlwgt $\leq 34398$ and workclass $\leq 4.500$ then $>50 \mathrm{~K}$ ( 1 / $14 / 0$ )

if fnlwgt $\leq 90725.500$ and fnlwgt > 65357 and hours-per-week $\leq 41$ then $<=50 \mathrm{~K} \quad(13 / 2 / 0)$

if relationship $>1.500$ and hoursper-week $\leq 49$ and occupation $\leq 6.500$ and fnlwgt $\leq 273425$ and age $\leq 35.500$ and fnlwgt $>157517$ then $>50 \mathrm{~K} \quad(1$ / 14 / 0)

if relationship $\leq 1.500$ and marital-status $\leq 2$ and sex $>1.500$ and hours-per-week $\leq 47.500$ then $<=50 \mathrm{~K} \quad(19 / 1 / 0)$

if relationship > 1.500 and age > 47.500 and occupation $>5.500$ and fnlwgt > 173487 and education $\leq$ 
5.500 and age $\leq 57.500$ then $>50 \mathrm{~K}$ $(3 / 18 / 0)$

if fnlwgt $\leq 205814$ and age $>60.500$ then >50K $(5 / 17 / 0)$

if age > 44.500 and occupation > 3 and fnlwgt > 163993 and hours-perweek $>44.500$ and fnlwgt $\leq 344657$ then $<=50 \mathrm{~K}(21 / 5 / 0)$

if fnlwgt $>90725.500$ and hoursper-week > 39 and age > 48.500 and fnlwgt $\leq 272693.500$ and hours-perweek $\leq 49$ and race $\leq 1.500$ and age $\leq 54.500$ then >50K $(2 / 16 / 0)$ if relationship $\leq 1.500$ and education-num $\leq 12.500$ then $<=50 \mathrm{~K}$ $(8 / 0 / 0)$

if age > 44.500 and workclass s 3.500 and fnlwgt $>102468$ and occupation $\leq 1.500$ then $<=50 \mathrm{~K} \quad$ ( 9 / 1 / 0)

if fnlwgt > 265806 and fnlwgt $\leq$ 313666 and hours-per-week $\leq 65$ and age $\leq 44.500$ then >50K (1/17/ 0)

if hours-per-week $\leq 47$ and fnlwgt > 140014 and fnlwgt $\leq 200941.500$ and fnlwgt $\leq 168774$ and occupation > 6.500 then $<=50 \mathrm{~K} \quad(7 / 1 / 0)$

if fnlwgt > 208415.500 and fnlwgt s 316152 and workclass $\leq 3.500$ and age $\leq 44.500$ and fnlwgt $>245527.500$ then $\quad<=50 \mathrm{~K} \quad(15 / 1 / 0)$

if age $\leq 44.500$ and age $>36.500$ and fnlwgt > 76409 and fnlwgt $\leq 120762$ and hours-per-week $\leq 43.500$ then $>50 \mathrm{~K}(2 / 18 / 0)$

if hours-per-week $\leq 47$ and sex $\leq$ 1.500 and relationship $\leq 1.500$ and age $>49$ then $<=50 \mathrm{~K}(6 / 0 / 0)$ if hours-per-week $>47$ and fnlwgt > 156146 and occupation $>6.500$ then $>50 \mathrm{~K}(1 / 8 / 0)$

if fnlwgt $\leq 116039$ and fnlwgt > 110042 and fnlwgt $\leq 112875$ then $<=50 \mathrm{~K} \quad(7 / 1 / 0)$

if fnlwgt $\leq 101759.500$ and fnlwgt > 50606 and age $>51$ then $<=50 \mathrm{~K}$ ( 5 / 0 / 0 )

if hours-per-week > 47 and fnlwgt > 171588 and native-country $\leq 5.500$ and fnlwgt $>349186$ then >50K (1 / $12 / 0)$

if age $\leq 47.500$ and education $\leq$ 1.500 and age $>43.500$ and fnlwgt > 85717 and workclass $\leq 5.500$ and marital-status $>1.500$ then $<=50 \mathrm{~K}$ $(15 / 1 / 0)$

if workclass > 3.500 and fnlwgt s 326967 and fnlwgt $>169132.500$ and age > 36.500 then >50K (3/ 13/ 0) if race > 1.500 and workclass > 2.500 and fnlwgt > 118569.500 and fnlwgt $\leq 337590.500$ then $\quad<=50 \mathrm{~K} \quad$ (9 ( 0 / 0 )

if hours-per-week $>47$ and fnlwgt $\leq$ 215753 and age $\leq 41.500$ and age > 33.500 and education $>2.500$ then $>50 \mathrm{~K}(0 / 8 / 0)$

if fnlwgt $\leq 99382$ and age $\leq 37.500$ and fnlwgt $>56670.500$ then $<=50 \mathrm{~K}$ $(5 / 0 / 0)$

if education $\leq 1.500$ and age > 43.500 and fnlwgt $\leq 363603$ and fnlwgt $>199825.500$ and age $>53.500$ then >50K $(0 / 7 / 0)$

if fnlwgt $>150593.500$ and maritalstatus $\leq 2.500$ and fnlwgt $\leq$ 156088.500 then $<=50 \mathrm{~K}$ ( $5 / 0$ / 0) if fnlwgt $\leq 157065$ and fnlwgt > 90725.500 and occupation $\leq 5$ and hours-per-week $\leq 47.500$ and age $\leq$ 59.500 then $>50 \mathrm{~K}(3 / 17 / 0)$

if hours-per-week $\leq 47$ and fnlwgt $\leq$ 343569.500 and fnlwgt $>102468$ and fnlwgt $\leq 150330$ then $\quad<=50 \mathrm{~K} \quad(10$ / $1 / 0)$

if fnlwgt > 205949 and education > 5.500 and occupation $>3$ then $<=50 \mathrm{~K}$ ( $9 / 1 / 0)$

if hours-per-week > 46.500 and fnlwgt > 99382 and fnlwgt $\leq$ 181590.500 and hours-per-week > 51 and occupation $>3$ then $>50 \mathrm{~K}$ ( $3 /$ $13 / 0)$

if fnlwgt $\leq 343569.500$ and hoursper-week $\leq 46.500$ and occupation $\leq$ 8.500 and fnlwgt $>174586$ and hoursper-week $>37$ and fnlwgt $\leq 203880$ then $<=50 \mathrm{~K} \quad(10 / 2 / 0)$

if hours-per-week $\leq 58$ and workclass $\leq 3.500$ and fnlwgt > 170125 and fnlwgt $\leq 181590.500$ and hours-per-week > 32.500 then >50K $(0 / 7 / 0)$

if hours-per-week $>57.500$ and age $\leq 47.500$ then $<=50 \mathrm{~K} \quad(10 / 2 / 0)$ if workclass $\leq 4.500$ and age > 33.500 and fnlwgt $>234132$ and education $>1.500$ then $>50 \mathrm{~K}$ (2 / $9 / 0)$

if fnlwgt > 165376.500 and fnlwgt s 197037 then $<=50 \mathrm{~K}(7 / 1 / 0)$

if hours-per-week > 53.500 and relationship > 1.500 then $>50 \mathrm{~K} \quad(0$ / 6 / 0)

if relationship $\leq 1.500$ and education $>2.500$ and hours-perweek $>37.500$ then $<=50 \mathrm{~K}$ ( 8 / 0 / $0)$ 
if fnlwgt $\leq 370677$ and sex $>1.500$ and fnlwgt $\leq 335257$ then $>50 \mathrm{~K}$ (1 / $11 / 0)$

if age $>50.500$ then $<=50 \mathrm{~K} \quad(6 / 1$ / 0$)$

if hours-per-week $\leq 42.500$ and occupation $\leq 8.500$ and educationnum $\leq 13.500$ and fnlwgt $>416296$ then $<=50 \mathrm{~K}(4 / 0 / 0)$

if age $>37.500$ and age $\leq 40.500$ and fnlwgt $\leq 182884$ then $>50 \mathrm{~K} \quad(0 / 6$ ( 0 )

if fnlwgt $\leq 102068.500$ and age $\leq$ 43.500 and age $>36$ then $<=50 \mathrm{~K} \quad(7$ / 0 / 0 )

if age > 42.500 and workclass $\leq$ 4.500 and age $>45.500$ then $>50 \mathrm{~K}$ $(1 / 8 / 0)$

if age $>45.500$ then $<=50 \mathrm{~K}$ ( $4 / 0$ ( 0 )

Nilai uji dataset menggunakan algoritma neural network didapatkan data akurasi yang terlihat pada gambar 3.2. sebagai berikut:

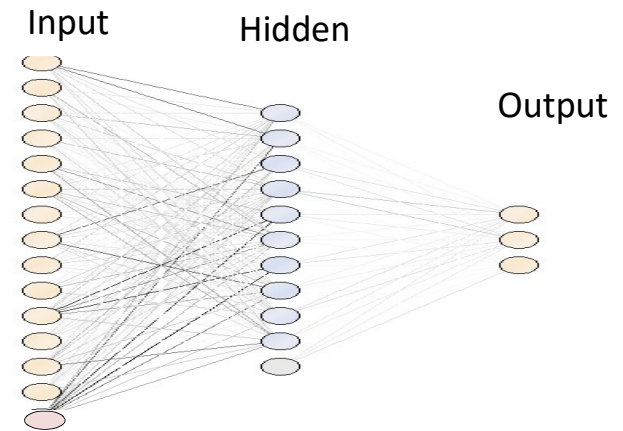

Gambar 3.2. Arsitektur Jaringan yang didapat dari Hasil Eksperimen Neural Network

Pada gambar 3.2. terlihat 10 buah hidden layer dan 1 buah Threshold untuk mendapatkan nilai akurasi sebesar $83.62 \%$ pada algoritma Neural Network dengan 3 buah output yang akan dihasilkan dan 14 buah input yang didapat dari atribut age, workclass, fnlwgt, education, education-num, marital-status, occupation, relationship, race, sex, capitalgain, capital-loss, hours-per-week, nativecountry dan 1 buah Threshold. Nilai dari setiap Node sebagai berikut:

Output

Class $<=50 \mathrm{~K}$ (Sigmoid)

Node 1: 2.202

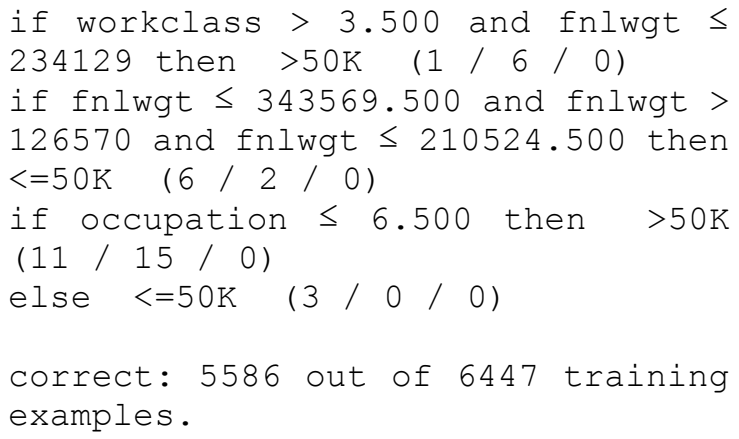

Sedangkan pada Neural Network Parameter penunjang seperti penentuan training cycle, learning rate, dan momentum sangat berpengaruh terhadap akurasi yang akan dihasilkan pada eksperimen yang dilakukan. Setelah itu akan muncul nilai akurasi, precision, recall, AUC (Optimistic), AUC, AUC (Pessimistic) yang didapat dari hasil eksperimen yang telah dilakukan.

Node 2: 1.918

Node 3: 5.125

Node 4: 8.848

Node 5: 3.301

Node 6: 1.703

Node $7:-4.852$

Node 8: 2.791

Node 9: 5.925

Node 10: 3.626

Threshold: -3.293

Class $>50 \mathrm{~K}$ (Sigmoid)

Node 1: -2.202

Node 2: -1.918

Node 3: -5.126

Node 4: -8.849

Node 5: -3.301

Node 6: -1.703

Node $7: 4.853$

Node 8: -2.791

Node 9: -5.925

Node 10: -3.626

Threshold: 3.293

Class $>=50$ (Sigmoid)

Node 1: -0.929

Node 2: -1.012

Node 3: -0.978

Node 4: -0.935

Node 5: -1.827

Node 6: -1.184

Node $7:-1.146$

Node 8: -1.013

Node 9: -0.962

Node 10: -0.899

Threshold: -5.399 


\begin{tabular}{|c|c|c|c|c|}
\hline \multicolumn{5}{|c|}{ (0) TableView Oplotview } \\
\hline \multicolumn{5}{|c|}{ 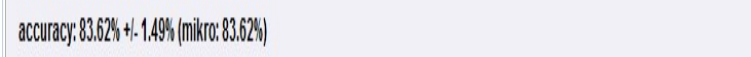 } \\
\hline & toe $<=50 \mathrm{~K}$ & tove $550 \mathrm{~K}$ & tove $x=50$ & dass precision \\
\hline pred. $<=50 \mathrm{~K}$ & 440 & 679 & 1 & $86.00 \%$ \\
\hline pred. $350 \mathrm{~K}$ & 376 & 922 & 0 & $71.03 \%$ \\
\hline pred $x=50$ & 0 & 0 & 0 & $0.00 \%$ \\
\hline dass recall & $9224 \%$ & $57.59 \%$ & $0.00 \%$ & \\
\hline
\end{tabular}

\section{Gambar 3.4 Hasil Akurasi Neural Network}

Nilai akurasi yang didapatkan dari hasil prediksi perhitungan menggunakan algoritma Neural Network terhadap data pendapatan rumah tangga sesuai dengan gambar 3.4. Dengan perhitungan akurasi sebagai berikut:

$$
\begin{aligned}
& \text { Accuracy }=\frac{4470+922}{4470+679+376+922} \\
& =0.83 \\
& \text { Precision }=\frac{922}{376+922}=0.71 \\
& \text { Recall }=\frac{922}{679+922}=0.57 \\
& F-\text { Measure }=\frac{2 * 0.57 * 0.71}{0.57+0.71}=0.63
\end{aligned}
$$

Selanjutnya akan didapatkan hasil dari confusion matriks yaitu pada gambar 3.5. sebagai berikut :

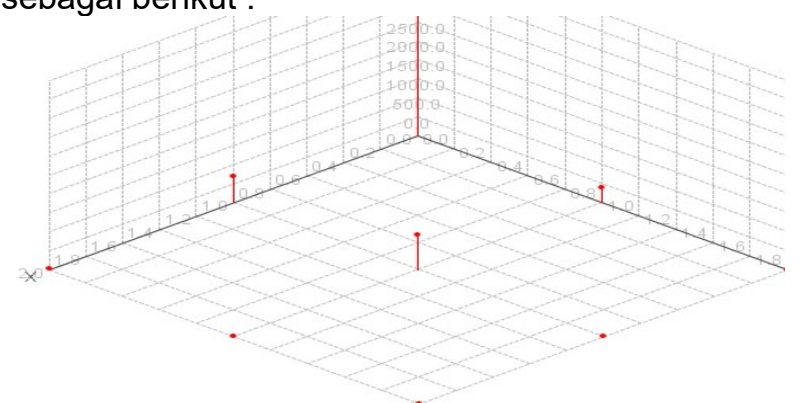

\section{Gambar 3.5 Hasil Confusion matrix pada akurasi $\mathbf{8 3 . 6 2 \%}$ dengan Rapid Miner}

Hasil Confusion Matrix pada gambar 3.5 dari nilai akurasi Neural network sebesar $83.62 \%$ menunjukan bahwa nilai output berupa

\section{Referensi}

F. Nurwanto, I. Ardiyanto and S. Wibirama, "Light sport exercise detection based on smartwatch and smartphone using kNearest Neighbor and Dynamic Time nilai threshold untuk pendapatan kurang dari sama dengan $\$ 50.000$ sebesar -3.293 , dan untuk output pendapatan yang lebih besar dari $\$ 50.000$ nilai threshold sebesar 3.293 sedangkan untuk output pendapatan yang lebih besar sama dengan $\$ 50.000$ nilai threshold sebesar -5.399 .

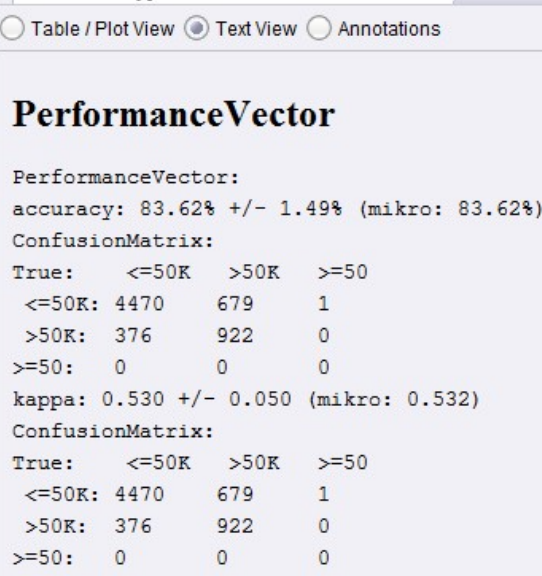

Gambar 3.6. hasil Performance Vector Neural Network pada data pendapatan rumah tangga dengan Rapid Miner

Gambar 3.6. menunjukkan performance vector dari data pendapatan rumah tangga menggunakan algoritma neural network yang menunjukkan nilai akurasi sebesar $83.62 \%$ serta penjabaran nilai untuk confusion matrix.

\section{Kesimpulan dan Saran}

Penelitian ini dilakukan komparasi antara K-nearest neigbour dengan algoritma neural network yang akan menghasilkan nilai akurasi pada data pendapatan rumah tangga dibeberapa Negara didunia sebesar $83.62 \%$ pada neural network dan akurasi sebesar $70,49 \%$ pada algoritma K-Nearest neighbor dengan demikian dapat dipastikan neural network dapat lebih tepat dalam memprediksi data pada pengolahan dataset pendapatan rumah tangga yang diambil dari $\mathrm{UCl}$ dataset pada Data sensus pendapatan yang dilakukan oleh Ronny Kohavi and Barry Becker pada sensu Beureu.

Warping algorithm," 2016 8th International Conference on Information Technology and Electrical Engineering (ICITEE), Yogyakarta, 2016, pp. 1-5.

Gorunescu, Florin. (2011). Data Mining: Concepts, Models and Techniques. 
Verlag Berlin Heidelberg, Springer. Jerman.

Guillet, Fabrice. Hamilton, Howard J. (2007). Quality Measures in Data Mining. Verlag Berlin Heidelberg, Springer. Jerman.

Han,J\& Kamber, Micheline. (2007). Data Mining Concepts, Models and Techniques. Second Edition, Morgan Kaufmann Publisher. Elsevier.

Heaton, Jeff. (2010). Programming Neural Networks With Encog 2 In Java. Heaton Research.Inc, USA.

Larose, D. (2005). Discovering Knowledge in Data. New Jersey, John Willey \& Sons.Inc.

Liao, Warren. T. \& Triantaphyllou.Evangelos. (2007). Recent Advances in Data Mining of Enterprise Data: Algorithms and Applications. Series: Computer and Operation Research. 6. 190.

Lim TS, Loh WY, Shih YS.(1999). A comparison of prediction accuracy, complexity, and training time of thirtythree old and new classification algorithms. Kluwer Academic Publishers: Boston.

Maimon, Oded\& Rokach, Lior. (2010). Data Mining and Knowledge Discovery Handbook, Springer, New York.

Mihov, Valentin. (2015). Adult Income Data Set Analysis with IPython. Sofia University, Bulgaria.

Min, Hui Tsai, et al.(2010). Profiling U.S. Household Income. https://faculty.biu.ac.il/ yahavi1/Projects /CP2010T1 rep.pdf.

Myatt, Glenn J. (2007). Making sense of data : A Practical Guide to Exploratory data analysis and Data Mining. John Wiley \& Sons Inc, New Jersey.

Priyanti, Evy.(2017).Implementasi Neural Network pada prediksi pendapatan rumah tangga. AMIK BSI. Swabumi.

Shukla, Anupam. Tiwari, Ritu. \& Kala, Rahul. (2010). Real Life Application of Soft Computing.New York: Taylor and Francis Groups, LLC.

Siang, Jong Jek (2009). Jaringan Syaraf Tiruan dan Pemrogramannya menggunakan MATLAB. Penerbit Andi. Yogjakarta.

Singh Asmita, Malka N. Halgamuge, Rajasekaran Lakshmiganthan. (2017).Impact of Different Data Types on Classifier Performance of Random Forest, Naïve Bayes, and K-Nearest Neighbors Algorithms. School of Computing and Mathematics Charles Sturt University Melbourne, Australia. (IJACSA) International Journal of
Advanced Computer Science and Applications

S. S. Nikam. A Comparative Study of Classification Techniques in Data Mining Algorithms. Orient.J. Comp. Sci. and Technol; 8(1), April 2015

Topiwalla, Mohammed. (2013). Machine Learning on $\mathrm{UCl}$ Adult data set using various classifier algoritms and scaling up the accuracy using extreme gradient boosting. University of SP Jain School of Global Management.

Vercellis,C. (2009). Business Intelligence: Data Mining and Optimization for Decision Making. Wiley.

Witten,I. Frank, E., \& Hall. (2011). Data Mining: Practical Machine Learning and tools. Morgan Kaufmann Publisher, Burlington. 